\title{
Management of bronchiectasis and chronic suppurative lung disease in Indigenous children and adults from rural and remote Australian communities
}

\author{
Anne B Chang, Keith Grimwood, Graeme Maguire, Paul T King, Peter S Morris and Paul J Torzillo
}

T he burden of respiratory disease among Indigenous Australians remains high. Death rates from respiratory disease (excluding lung cancer) in adults are 5-6 times higher than in non-Indigenous Australians. ${ }^{1}$ In a 2004-05 national survey, 31\% of Indigenous Australians self-reported long-term respiratory problems. ${ }^{2}$ The Australia-wide prevalence of bronchiectasis in the Indigenous population is unknown, but it is disproportionately high in rural and remote Indigenous communities. The prevalence in Central Australia of at least 147 cases per 10000 Indigenous children $^{3}$ is significantly higher than the prevalence of cystic fibrosis (CF) in Australia as a whole (3.5 cases per 10000 children). ${ }^{4}$

People with bronchiectasis have a more rapid decline in lung function $^{5}$ and a reduced life expectancy. ${ }^{6}$ In a Central Australian study, hospitalised adults with bronchiectasis were shown to have seriously impaired lung function (mean forced expiratory volume in 1 second $\left[\mathrm{FEV}_{1}\right], 36 \%$ of predicted value), despite their relatively young age ( $23 \%$ were aged $<30$ years). ${ }^{7}$ Furthermore, the effects of bronchiectasis extend beyond the respiratory system ${ }^{8}$ - for example, it is an independent risk factor for atherosclerosis. ${ }^{9}$ There is evidence that effective management of bronchiectasis improves wellbeing and reduces morbidity. ${ }^{10}$

Despite its relatively frequent occurrence in Indigenous people, bronchiectasis is often undiagnosed. Moreover, its management ranges from symptomatic treatment alone (in the belief that little further can be done) to intensive management. In our previous position statement we focused on bronchiectasis in Indigenous children. ${ }^{4}$ Here we update the evidence and produce revised consensus recommendations for the management of bronchiectasis in both Indigenous adults and children from rural and remote Australian communities. Our methods are explained in Box 1, a summary of the evidence is presented in Box 2, and a concise list of the recommendations appears in Box 3. Each recommendation is discussed in more detail in the following text.

In drafting the recommendations, we recognised regional and individual heterogeneity. Our latest position statement is not intended for tertiary management. It is intended to provide a framework for care and not to replace the clinical assessment and judgement required for treating individual patients.

\section{Objectives of our position statement}

- To increase awareness of bronchiectasis in Indigenous children and adults and improve detection rates and management.

- To develop a national consensus for managing bronchiectasis in Indigenous children and adults living in rural and remote Australian communities.

\section{Case definition of bronchiectasis and chronic suppurative lung disease}

In the past, bronchiectasis was defined by postmortem histopathology. Later, bronchograms became the gold standard for

\section{ABSTRACT}

- Consensus recommendations for managing bronchiectasis in Indigenous children and adults living in rural and remote regions were developed during a multidisciplinary workshop and were based on available systematic reviews.

- Successful diagnosis, management and prevention of bronchiectasis in Indigenous Australians requires access to comprehensive health care services, as well as improved housing, education and employment and reduced poverty levels.

- Diagnosis of bronchiectasis requires a chest high-resolution computed tomography scan. Children who have bronchiectasis symptoms but non-diagnostic scans are described as having chronic suppurative lung disease (CSLD), rather than bronchiectasis. Untreated CSLD may progress to bronchiectasis.

- Chronic wet cough ( $>4$ weeks) or recurrent wet cough (>2 episodes/year) are important but often under-reported symptoms. Bronchiectasis is suspected when chronic cough is excessively prolonged (>12 weeks) or if a chest radiographic abnormality persists despite appropriate therapy.

- Intensive treatment aims to improve symptom control and quality of life while preserving lung function and reducing acute exacerbation frequency.

- Antibiotics should be prescribed for acute infective episodes according to culture results of respiratory secretions, local susceptibility patterns and clinical severity. Patients not responding promptly to oral antibiotics should be hospitalised for more intensive treatment.

- Ongoing care requires regular primary health care and specialist review, including monitoring for complications and comorbidities. Corticosteroids, bronchodilators and mucoactive agents may be used in individual cases, but routine use is not recommended. Physiotherapy and exercise should be encouraged, nutrition optimised, environmental pollutants (including tobacco smoke) avoided, and immunisations maintained.

MJA 2008; 189: 386-393
Abbreviations
CF Cystic fibrosis
C-HRCT Chest high-resolution computed tomography
COPD Chronic obstructive pulmonary disease
CSLD Chronic suppurative lung disease
$\mathrm{FEV}_{1} \quad$ Forced expiratory volume in 1 second
RCT Randomised controlled trial 
diagnosis before being replaced by chest high-resolution computed tomography (c-HRCT) scans. Currently, bronchiectasis, defined in terms of "irreversible dilatation of peripheral airways", is diagnosed by c-HRCT scans. ${ }^{16}$ The diagnostic radiographic criteria are described elsewhere. ${ }^{16}$ In children, this radiology-based definition poses problems, for several reasons: ${ }^{15,17}$

- Extrapolating c-HRCT findings of bronchiectasis from adult studies may be inappropriate for children, as morphology of the airway and lungs changes with age;

- At least two c-HRCT scans (separated by an undefined period) are required to confirm irreversible airway dilatation. For Indigenous people from remote communities, this poses logistic difficulties; and

- c-HRCT scans performed in different clinical states yield different results.

Instead, paediatricians prefer to use the term chronic suppurative lung disease (CSLD) to describe the condition of children with symptoms of bronchiectasis (Box 4) but without confirmatory c-HRCT findings. This assumes there is a continuum of potentially reversible to irreversible airway injury following repeated infection and that aggressive management of children with CSLD may prevent bronchiectasis. ${ }^{15}$ The management of CSLD and bronchiectasis is the same. In contrast, adults with chronic cough and sputum production without evidence of bronchiectasis on c-HRCT scan may have chronic obstructive pulmonary disease (COPD) (if airflow obstruction is demonstrated on spirometry) or chronic bronchitis.

\section{Recommendation 1}

1a. CSLD describes respiratory symptoms and signs (Box 4) in children without radiographic features of bronchiectasis.

1b. Chest HRCT scans are needed to confirm bronchiectasis as a diagnosis.

Agreement level: A+, 86\%; A, 14\%

Grade: not applicable; evidence level: cohort studies

\section{Investigations}

\section{Radiology}

Plain chest x-rays are insensitive for diagnosing bronchiectasis. Chest HRCT scans are performed when radiographic changes of pneumonia persist ${ }^{18}$ despite adequate treatment or when symptoms suggest bronchiectasis (Box 4). In children, c-HRCT scans should only be conducted after specialist review, in view of the frequent need for anaesthesia and the greater relative radiation dose.

\section{Recommendation 2}

Patients with symptoms or signs of bronchiectasis need a cHRCT scan to confirm the diagnosis and to assess the severity and extent of disease. Specialist advice is required before ordering scans for children.

Agreement level: A+, 95\%; A, 2.5\%; A-, 2.5\%

Grade: moderate; evidence level: cohort studies

\section{Aetiology}

Patients with bronchiectasis are investigated for possible aetiology (Box 4), as there is evidence that this can influence management and severity. ${ }^{19}$ However, even with extensive investigation, a cause

\section{Methods}

The consensus group comprised 37 representatives from Aboriginalcontrolled community health organisations, remote health services, medical schools, research institutes and primary, secondary and tertiary health centres. Collectively, the group possessed expertise in Indigenous primary health care; paediatric and adult respiratory medicine; microbiology; paediatric and adult infectious diseases; tobacco control; rural and remote health; and public health.

One of the authors (A B C) performed an updated search (from a previous search in March $2006^{8}$ ) of the PubMed and Cochrane Central Library databases using the text-words "bronchiectasis" or

"suppurative lung disease" and "controlled trials". Only full articles published in English were retrieved. Recommendations were formed based on the quality of supporting evidence using the GRADE system: ${ }^{11}$

- High: further research is very unlikely to change our confidence in the estimate of effect;

- Moderate: further research is likely to have an important impact on our confidence in the estimate of effect and may change the estimate;

- Low: further research is very likely to have an important impact on our confidence in the estimate of effect and is likely to change the estimate; or

- Very low: any estimate of effect is very uncertain.

As relative risk or estimate of effect size were available in very few of the articles, the decision to upgrade a recommendation was primarily based on the likelihood of whether further research would have an effect on the recommendation. The grade was assessed by three authors ( $A B C, K G, P J T)$. We used a modified Delphi process to reach consensus on the recommendations based on a similar model described by Vakil et al. ${ }^{12}$

Each recommendation was discussed during a 2-day workshop held in Darwin (24-25 Nov 2007). When disagreements occurred, the recommendation was rephrased by workshop attendees $(n=22)$, and these were voted on by all in the consensus group (a panel of speakers and others with relevant expertise but who were unable to attend the workshop). The document was also circulated more widely, but only those who responded (see below) were included in the list.

Agreement with a statement ( $A+, A$, or $A-$ ) by two-thirds (ie, $\geqslant 67 \%$ ) of the group was defined a priori as consensus. For the final

recommendation, a 6-point Likert scale was used: $A+=$ agree strongly; $A=$ agree with minor reservation; $A-=$ agree with major reservation; $\mathrm{D}-=$ disagree with major reservation; $\mathrm{D}=$ disagree with minor reservation; $\mathrm{D}+=$ disagree strongly.

\section{Consensus group members}

Adelaide: Ral Antic, ${ }^{*}$ Lloyd Einsiedel; ${ }_{i}^{\dagger}$ Alice Springs: Andrew White, ${ }^{\dagger}$ Stephen Brady, ${ }^{\dagger}$ Erik Tikoft, ${ }^{\dagger}$ Emma Tilley, ${ }^{\dagger}$ Carmel Hattch, ${ }^{\star}$ Paul Torzillo; ${ }^{\dagger}$ Brisbane: Scott Bell, ${ }^{\star}$ Keith Grimwood, ${ }^{\dagger}$ Patricia Valery, ${ }^{\dagger}$ Brent Masters; ${ }^{\dagger}$ Cairns: Graeme Maguire; ${ }^{\dagger}$ Darwin: Malcolm MacDonald, ${ }^{\dagger}$ Peter Morris, ${ }^{\dagger}$ Amanda Leach, ${ }^{\dagger}$ Keith Edwards, ${ }^{\dagger}$ Jonathan Carapetis, ${ }^{\dagger}$ Gabrielle McCallum, ${ }^{\dagger}$ Alan Ruben, ${ }^{\dagger}$ David Thomas, $^{\dagger}$ Anne Chang, ${ }^{\dagger}$ Bart Currie, ${ }^{*}$ Allen Cheng, ${ }^{*}$ Jo Wright, ${ }^{*}$ Annie Whybourne,* Louise Martin, * Paul Bauert, * Carolyn Maclennan, ${ }^{*}$ Ngiare Brown; ${ }^{\star}$ Melbourne: Paul King, ${ }^{\dagger}$ Kim Mulholland, ${ }^{*}$ Colin Robertson, ${ }^{\star}$ Sarath Ranganathan; ${ }^{*}$ Perth: Deborah Lehman, ${ }^{\dagger}$ Lou Landau; * Sydney: Paul Torzillo, ${ }^{\dagger}$ Peter Van Asperen. *

*Voted on recommendations. †Attended workshop.

is often not found. ${ }^{20}$ In an Australian study of non-Indigenous adults with newly diagnosed bronchiectasis, ${ }^{20}$ a specific cause was identified in only $27 / 103$ patients (26\%). In another study, 8/65 Indigenous children with bronchiectasis (12\%) had an underlying contributing factor. ${ }^{3}$ 


\section{Possible interventions for management of chronic suppurative lung disease (CSLD)}

\section{Evidence type/study}

Antimicrobials (by type)

General

Macrolides $^{\dagger 13}$

Cochrane review, other systematic review*

RCT and reviews ${ }^{\star 14}$ conducted over 2-6 months

Nebulised tobramycin* ${ }^{\star \dagger}$ Double-blind crossover RCT in 30 patients with PsA, 6 months each

\section{Antimicrobials (by time)}

Short-term (<1 month)

Medium-term

(1-11 months)

Long-term RCTs*

( $\geqslant 12$ months)
Multiple cohort studies*

Cochrane review, other systematic reviews ${ }^{\star 14}$

\section{Anti-inflammatories}

Oral NSAIDs ${ }^{\S}$

Inhaled indomethacin

\section{Mucolytics}

Bromhexine

rhDNase

\section{Airway clearance}

Chest physiotherapy

Inhaled hyperosmolar

agents

\section{Cochrane review*}

RCT in 25 adults, some with CSLD*

Cochrane review*

Systematic review*

\section{Cochrane review*}

Cochrane review, ${ }^{*}$ additional RCT

(non-blinded) using $7 \%$ hypertonic saline*
Summary of results

Generally beneficial. See text

Exacerbations significantly reduced in treatment arm, with reduction in sputum and symptoms and PFT improvement in some patients ${ }^{14}$

\section{Asthma therapies \\ Inhaled corticosteroids ${ }^{\star \star}$ Cochrane review* and other RCTs (see text)}

No significant effect of ICSs in Cochrane review. * Additional RCTs showed some benefit. Reduced exacerbation rate only seen in patients with PsA.*

Oral corticosteroids
Anticholinergics
${ }^{\dagger \dagger}$
$\beta_{2}$-Agonists $^{\dagger \dagger}$
LTRAs $^{\dagger \dagger}$

Cochrane review*

No RCTs

Cochrane review* No RCTs $^{\star}$

Cochrane review* ${ }^{*} \quad$ No RCTs

Cochrane review* No RCTs

Physical training

Cochrane review* and RCT,* which was

Pulmonary rehabilitation improves exercise tolerance; no additional included in Cochrane database as an abstract ${ }^{\ddagger \ddagger}$ advantage of simultaneous inspiratory muscle training

$\begin{array}{ll}\text { Oxygen (domiciliary) } & \text { No data on use as sole therapy* } \\ \text { Surgery } \S \S & \text { Cochrane review }\end{array}$

Consider data from COPD studies showing benefit in survival*

No RCTs. Cohort studies suggest benefit in selected cases*

\section{Vaccines}

Pneumococcal 23ๆๆ

Cochrane review*

No RCTs

Influenza

Cochrane review*

No RCTs

Acupuncture

$\mathrm{RCT}$ *

Improvement in QOL but not in sputum or 6-minute walking test

Model of follow-up

Nurse-led

Cochrane review*

No difference in exacerbations but increase in hospitalisations in nurseled care compared with doctor-led care ${ }^{\star \star \star}$

COPD = chronic obstructive pulmonary disease. $\mathrm{FEV}_{1}=$ forced expiratory volume in 1 second. ICS = inhaled corticosteroid. LTRA = leukotriene receptor antagonist. NSAID = non-steroidal anti-inflammatory drug. $\mathrm{QOL}=$ quality of life. $\mathrm{PFT}=$ pulmonary function test. PsA = Pseudomonas aeruginosa. $\mathrm{RCT}=$ randomised controlled trial. rhDNase = recombinant human deoxyribonuclease.

*For references, see Chang et al..$^{15} \dagger$ Antimicrobial resistance is a concern. ‡ Nebulised tobramycin is poorly tolerated in some patients. § In a cohort study, indomethacin $25 \mathrm{mg}$ three times a day for 28 days reduced neutrophil chemotaxis, but there was no change in levels of sputum albumin, elastase or myeloperoxidase (see Chang et a ${ }^{15}$ for reference). 9 Not universally available. ** Limited applicability in children (high-dose ICSs unsuitable for children, and children less likely to have PsA infections). †† No other data found by a single-reviewer search of PubMed and Cochrane Library databases in Oct 2007. 㧊 The data in the abstract that was in the Cochrane review were different from the actual results in the article. $\S \S$ Reduction in exacerbation rate similar in medically treated group. For adverse events of surgery, see Chang et al. ${ }^{15}$ ๆी Advocated, as vaccines reduce respiratory infections. ${ }^{\star \star \star}$ Increased health care cost implications. 


\section{Summary of consensus recommendations for bronchiectasis and chronic suppurative lung disease (CSLD)}

1a. CSLD describes respiratory symptoms and signs (Box 4) in children without radiographic features of bronchiectasis.

1b. Chest HRCT scans are needed to confirm bronchiectasis as a diagnosis.

2. Patients with symptoms or signs of bronchiectasis need a c-HRCT scan to confirm the diagnosis and to assess the severity and extent of disease. Specialist advice is required before ordering scans for children.

3. Perform baseline investigations. These include:

$>$ Culturing airway secretion;

$>$ Spirometry (when aged $>6$ years);

$>$ Immune function tests (Box 4);

$>$ Bronchoscopy (for foreign body, airway abnormality and microbiological culture);

$>$ Echocardiogram in adults (on specialist recommendation in children); and

$>$ Other investigations (eg, sweat test, tests for tuberculosis, barium swallow) may be necessary in selected patients.

4. Intensive medical therapy optimises general wellbeing, symptom control, lung function and quality of life, as well as reducing exacerbation frequency.

5. Antibiotics (Box 5) are based on lower airway microbiology (sputum or bronchoalveolar lavage), local antibiotic susceptibility patterns and clinical severity.

6. In patients not needing hospitalisation for an acute exacerbation, oral antibiotics are prescribed for at least 10-14 days.

7. Patients failing to respond to oral antibiotics for an acute exacerba-tion are hospitalised for more intensive treatment, including intravenous antibiotics. This usually requires hospitalisation for at least 7 days.
8. Inhaled and oral corticosteroids should not be routinely prescribed and only used on an individual basis.

9. Inhaled bronchodilators should not be routinely prescribed and only used on an individual basis.

10. Recombinant human deoxyribonuclease is contraindicated in non-CF bronchiectasis.

11. At present, other mucoactive agents are not routinely recommended.

12. Airway clearance manoeuvres are recommended, and physiotherapists' advice should be sought. The technique and frequency of chest physiotherapy is individualised.

13. Adults with bronchiectasis and limited exercise tolerance should receive pulmonary rehabilitation.

14. Assess and optimise children's nutrition.

15. Promote smoking elimination, including second-hand smoke exposure.

16. Avoid biomass smoke exposure.

17. Regularly monitor for complications and comorbidities (Box 6). When present, these are managed following standard therapies and guidelines.

18. Ensure timely annual influenza immunisation and that pneumococcal vaccines are administered according to NHMRC and local guidelines.

19. Comprehensive health service delivery systems are essential for managing chronic conditions in rural and remote regions.

Management guidelines, clear referral systems and links with specialists will strengthen the capacity of health services to deliver high-quality care.

$\mathrm{CF}=$ cystic fibrosis. $\mathrm{C}-\mathrm{HRCT}=$ chest high-resolution computed tomography. $\mathrm{NHMRC}=$ National Health and Medical Research Council.

\section{Risk factors}

Initial triggers for bronchiectasis are unknown, but animal models suggest that both inadequate mucous clearance and persistent infection are necessary. ${ }^{21,22}$ Consistent with this hypothesis, recurrent, severe pneumonia is a risk factor for the development of bronchiectasis in Indigenous Australian children. ${ }^{23}$ In a Central Australian study, 20\% of Indigenous children hospitalised with lobar pneumonia had chronic respiratory illness at 12-month follow-up (most commonly, CSLD). ${ }^{18}$

\section{Severity}

Bronchiectasis is assessed by symptoms and signs, radiology and pulmonary function. Pulmonary function tests, like spirometry (which typically shows an obstructive pattern), are performed at each review. However, as a marker for disease severity, pulmonary function tests are relatively insensitive, especially in young children. ${ }^{3}$ With increasing age, $\mathrm{FEV}_{1}$ values in bronchiectasis patients decline more rapidly and are used as a prognostic marker. ${ }^{5,6}$ As pulmonary hypertension can complicate severe bronchiectasis, ${ }^{8}$ an echocardiogram is recommended for all adults and for children with advanced disease. Respiratory failure can occur in advanced bronchiectasis, in which case oximetry, arterial blood gas measurement and polysomnography are required to assess the need for domiciliary oxygen or noninvasive ventilatory support.

The most frequent respiratory pathogens in children with CSLD and bronchiectasis are Haemophilus influenzae, Streptococcus pneu- moniae, Moraxella catarrhalis and, occasionally, Staphylococcus aureus. $^{8}$ Adult patients with bronchiectasis are also subject to infection with these pathogens, as well as Pseudomonas aeruginosa (associated with advanced disease), ${ }^{8}$ Aspergillus and non-tuberculous mycobacteria. ${ }^{24,25}$

\section{Recommendation 3}

Perform baseline investigations. These include:

- Culturing airway secretion;

- Spirometry (when aged $>6$ years);

- Immune function tests (Box 4);

- Bronchoscopy (for foreign body, airway abnormality and microbiological culture);

- Echocardiogram in adults (on specialist recommendation in children); and

- Other investigations (eg, sweat test, tests for tuberculosis, barium swallow) may be necessary in selected patients.

Agreement level: A+, 84\%; A, 5\%; A-, 11\%

Grade: moderate; evidence level: cohort studies

\section{Management}

Although there is no definitive evidence to support an early treatment approach, it is generally accepted that patients who have CSLD and are thus at risk of bronchiectasis will benefit from early treatment. A longitudinal study has shown that patients with 


\section{Bronchiectasis: symptoms and signs, associated conditions and tests}

\section{Symptoms and signs of bronchiectasis}

Excessively prolonged ( $>12$ weeks) wet cough, exertional dyspnoea, asthma-like symptoms and recurrent chest infections. Clinical signs, which are often delayed, include growth failure, digital clubbing, chest wall deformity, hyperinflation, and adventitial sounds on chest auscultation. ${ }^{3}$ In advanced disease, chronic hypoxaemia and signs of pulmonary hypertension may be present. In children, triggers for referral to a specialist include more than two episodes ( $>4$ weeks) of chronic wet cough per year responding to antibiotics, and persistent chest radiographic abnormality. As cough is commonly underreported by Indigenous people, it is useful to obtain additional medical information from the local community (eg, clinic staff, carers, health workers, clinic notes) about the nature and duration of cough. ${ }^{4}$

\section{Possible aetiologies in bronchiectasis}

Congenital diseases (eg, cystic fibrosis, primary ciliary dyskinesia, $\alpha 1$-antitrypsin deficiency, tracheobronchomegaly, bronchomalacia); immunodeficiency (eg, associated with hypo- $\boldsymbol{\gamma}$-globulinaemia, neutrophil function abnormalities, HIV infection); aspiration (including foreign body); chronic infections (eg, tuberculosis, aspergillosis, non-tuberculous mycobacterial infections); chronic obstructive pulmonary disease; bronchiolitis obliterans; and systemic (eg, autoimmune) disorders.

\section{Recommended blood tests at baseline}

Basic immune function assessment: full blood count, including differential white blood cell count; HIV testing (in adults and at-risk children); levels of IgG (+ subclasses), IgA, IgM, and IgE; and antibody responses to vaccine protein and polysaccharide antigens. In selected situations, other investigations may be necessary (eg, neutrophil function studies and lymphocyte subsets). In Central Australia, HTLV-1 serology should also be included.

delayed diagnosis have poorer lung function than patients receiving regular antibiotics and physiotherapy. ${ }^{26}$

\section{Antibiotics}

We recommend treating severe or persistent exacerbations of bronchiectasis with intravenous antibiotics and physiotherapy in hospital. Short antibiotic courses of 10-14 days reduce symptoms and levels of inflammatory markers and improve quality of life. ${ }^{8}$ In contrast, maintaining antibiotic treatment for as long as 12 months confers only a small benefit. ${ }^{27}$ Nonetheless, prolonged courses of macrolide antibiotics are being used. ${ }^{14}$ Azithromycin treatment for 6-36 months in patients with CF initially improves lung function and quality-of-life scores while reducing exacerbation frequency. However, these benefits are not sustained, and development of antibiotic resistance is a matter of concern. ${ }^{28,29}$ Studies of macrolide use in patients with non-CF bronchiectasis are limited. ${ }^{14}$ Adults receiving twice-weekly azithromycin for 6 months have been shown to have reduced respiratory exacerbations and sputum volume. ${ }^{13}$ However, until longer-term trials are conducted, macrolides cannot be routinely recommended.

Ideally, antibiotics should be prescribed according to sputum culture and susceptibility results. Young children cannot usually expectorate sputum and, if a child is seriously ill or unresponsive to empirical antibiotics, a lower airway specimen can be collected by bronchoalveolar lavage. Adults with severe disease should initially receive antipseudomonal antibiotic therapy if cultures are unavailable.

\section{Recommendation 4}

Intensive medical therapy optimises general wellbeing, symptom control, lung function and quality of life, as well as reducing exacerbation frequency.

Agreement level: A+, $100 \%$

Grade: high; evidence level: cohort studies, Cochrane reviews, randomised controlled trials (RCTs)

\section{Recommendation 5}

Antibiotics (Box 5) are based on lower airway microbiology (sputum or bronchoalveolar lavage), local antibiotic susceptibility patterns and clinical severity.

Agreement level: A+, 86\%; A, 14\%

Grade: moderate; evidence level: cohort studies

\section{Recommendation 6}

In patients not needing hospitalisation for an acute exacerbation, oral antibiotics are prescribed for at least 10-14 days.

Agreement level: $A+, 89 \% ; A, 8 \% ; D, 3 \%$

Grade: low; evidence level: cohort studies

\section{Recommendation 7}

Patients failing to respond to oral antibiotics for an acute exacerbation are hospitalised for more intensive treatment, including intravenous antibiotics. This usually requires hospitalisation for at least 7 days.

Agreement level: A+, $89 \% ; A, 8 \% ; D, 3 \%$

Grade: low; evidence level: cohort studies

\section{Corticosteroids, bronchodilators and mucolytics}

When asthma-like symptoms exist, lung function deterioration is accelerated. ${ }^{6}$ Patients with either bronchodilator or airway hyperresponsiveness should receive $\beta_{2}$-agonists and inhaled corticosteroids. But reviews have found insufficient evidence to recommend their routine use. ${ }^{14,15}$ The trials involved small numbers of subjects and post-hoc subgroup analyses of doubtful validity. Although sputum volume was reduced in patients receiving inhaled corticosteroids, this required high doses, which were associated with adverse long-term complications.

At present there is insufficient evidence to recommend mucolytics (such as mannitol or hypertonic saline) as routine treatment. Recombinant deoxyribonuclease, although effective in patients with CF, is harmful to adults with bronchiectasis, as it is associated with higher exacerbation and hospitalisation rates and a more rapid decline in lung function. ${ }^{31}$

\section{Recommendation 8}

Inhaled and oral corticosteroids should not be routinely prescribed and only used on an individual basis.

Agreement level: A+, 95\%; A, 2.5\%; A-, 2.5\%

Grade: low; evidence level: Cochrane reviews, RCTs

\section{Recommendation 9}

Inhaled bronchodilators should not be routinely prescribed and only used on an individual basis.

Agreement level: A+, 92\%; A, 8\%

Grade: low; evidence level: Cochrane reviews 


\section{Recommended antibiotics for patients with bronchiectasis or chronic suppurative lung disease}

Mild-moderate exacerbation (oral therapy) Severe exacerbation (intravenous therapy)

\begin{tabular}{|c|c|c|}
\hline Initial empirical therapy* & Amoxycillin or doxycycline $^{\dagger}$ & Children: cefotaxime or ceftriaxone; adults: ticarcillin-clavulanate \\
\hline \multicolumn{3}{|l|}{ Organism-specific therapy } \\
\hline Haemophilus influenzae & Amoxycillin & Ampicillin \\
\hline$\beta$-Lactamase-positive organisms & Amoxycillin-clavulanate or doxycycline ${ }^{\dagger}$ & Cefotaxime or ceftriaxone \\
\hline Streptococcus pneumoniae & Amoxycillin & Benzylpenicillin G \\
\hline Moraxella catarrhalis & Amoxycillin-clavulanate & Cefotaxime or ceftriaxone \\
\hline Staphylococcus aureus & Flucloxacillin or cephalexin & Flucloxacillin \\
\hline Pseudomonas aeruginosa & Ciprofloxacin & $\begin{array}{l}\text { Children: ticarcillin-clavulanate + tobramycin; }{ }^{\ddagger} \text { adults: ticarcillin- } \\
\text { clavulanate }\end{array}$ \\
\hline $\begin{array}{l}\text { Methicillin-resistant } S \text {. aureus and } \\
\text { non-tuberculous mycobacteria }\end{array}$ & Seek specialist advice & Seek specialist advice \\
\hline
\end{tabular}

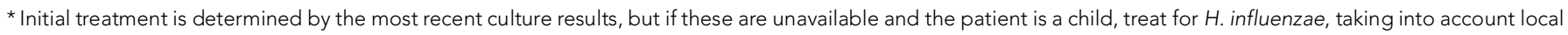

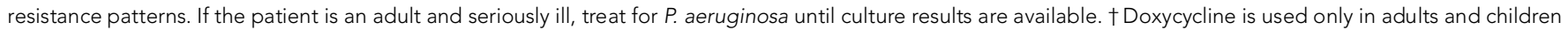

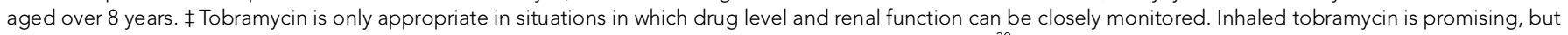
there is currently insufficient evidence for its routine use in patients with non-cystic fibrosis bronchiectasis. ${ }^{30}$

\section{Recommendation 10}

Recombinant human deoxyribonuclease is contraindicated in non-CF bronchiectasis.

Agreement level: A+, 95\%; A, 5\%

Grade: low; evidence level: Cochrane reviews

\section{Recommendation 11}

At present, other mucoactive agents are not routinely recommended.

Agreement level: A+, 86\%; A, 14\%

Grade: low; evidence level: Cochrane reviews

\section{Airway clearance}

Chest physiotherapy is standard treatment in $\mathrm{CF}$ and improves airway clearance. ${ }^{8}$ There has been little research on the effect of physiotherapy in patients with non-CF bronchiectasis. Positive expiratory pressure techniques are commonly used in children. Head-down tilt is avoided because it increases gastro-oesophageal reflux and possible aspiration. ${ }^{32}$ Various physiotherapy techniques with varying benefits have been employed. ${ }^{33}$

\section{Recommendation 12}

Airway clearance manoeuvres are recommended, and physiotherapists' advice should be sought. The technique and frequency of chest physiotherapy is individualised.

Agreement level: A+, 97\%; A, 3\%

Grade: moderate; evidence level: Cochrane review (no RCT), RCTs (for physiotherapy techniques)

\section{Recommendation 13}

Adults with bronchiectasis and limited exercise tolerance should receive pulmonary rehabilitation.

Agreement level: A+, 86\%; A, 8\%; A-, 3\%; D, 3\%

Grade: moderate; evidence level: Cochrane review

\section{Nutrition}

Evidence from patients with $\mathrm{CF}$ suggests that poor nutrition is a risk factor for respiratory decline. Nutritional status may have a greater impact on CSLD and bronchiectasis in a child with developing lungs than in an adult.

\section{Recommendation 14}

Assess and optimise children's nutrition.

Agreement level: A+, 97\%; A-, 3\%

Grade: moderate; evidence level: extrapolated from cohort studies on chronic lung disease

\section{Minimising further lung injury}

Environmental pollutants, including tobacco and indoor woodsmoke, exacerbate chronic respiratory illnesses by increasing the risk of acute respiratory infection. ${ }^{4}$ Efforts to reduce childhood smoke exposure, in utero and in the home environment, must be maximised.

\section{Recommendation 15}

Promote smoking elimination, including second-hand smoke exposure.

Agreement level: A+, $100 \%$

Grade: high; evidence level: extrapolated from cohort studies on chronic lung disease

\section{Recommendation 16}

Avoid biomass smoke exposure.

Agreement level: A+, 97\%; A, 3\%

Grade: low; evidence level: extrapolated from cohort studies on respiratory infection

\section{Monitoring, comorbidities and prevention of acute exacerbations}

Patients with bronchiectasis have increased incidence of comorbidities. ${ }^{8}$ Attention to oral hygiene is necessary, as dental caries are associated with pulmonary disease severity. ${ }^{8}$ In adults, bronchiectasis may coexist with COPD (in 29\%-50\% of patients) and is frequently undiagnosed. ${ }^{8,34}$

Although published data are limited, a coordinated management approach may reduce bronchiectasis mortality and complication 
rates. Patients with CSLD or bronchiectasis require regular primary health care, specialist reviews and intensive therapy to reduce exacerbation frequency and severity and to slow pulmonary decline. $^{26,35}$

\section{Recommendation 17}

Regularly monitor for complications and comorbidities (Box 6). When present, these are managed following standard therapies and guidelines.

Agreement level: A+, 95\%; A, 5\%

Grade: moderate; evidence level: cohort studies

\section{Public health issues, prevention and appropriate health care delivery}

Breastfeeding helps protect children from CSLD and bronchiectasis. ${ }^{23}$ All Indigenous children and adults should receive the routine vaccinations outlined in The Australian immunisation handbook, ${ }^{36}$ including annual influenza and pneumococcal vaccines. These and other important public health measures are beyond the scope of our article. In brief, health is closely linked to socioeconomic factors, and increased poverty is an independent risk factor for acute respiratory infection. ${ }^{4}$ Successful management and prevention of bronchiectasis in Indigenous people will only be achieved by delivering comprehensive health care accompanied by improvements in housing, education and employment and reduction in poverty levels.

Effective delivery of treatment and disease control programs requires a comprehensive primary health care service. Greater efforts are needed to educate all health care providers in the following areas:

- Identifying and referring children and adults with suspected CSLD or bronchiectasis; and

- Enhancing the role of health care providers in primary management of these disorders

Delivering optimal health care in a setting of entrenched poverty and major social disadvantage is difficult. However, the definite benefits of optimal care should not be underestimated. The challenge for health service systems is to find ways to deliver effective, quality health care despite problems such as remoteness, endemic poverty, educational disadvantage, dysfunctional communities, and comorbidities in children, their carers and adults. Priority areas for health delivery and research are outlined in Box 7 .

\section{Recommendation 18}

Ensure timely annual influenza immunisation and that pneumococcal vaccines are administered according to National Health and Medical Research Council (NHMRC) and local guidelines.

Agreement level: A+, 97\%; A, 3\%

Grade: low for pneumococcal vaccine, moderate for influenza vaccine; evidence level: Cochrane review (no RCT)

\section{Recommendation 19}

Comprehensive health service delivery systems are essential for managing chronic conditions in rural and remote regions. Management guidelines, clear referral systems and links with specialists will strengthen the capacity of health services to deliver high-quality care.

Agreement level: A+, 100\%

Grade: not applicable

\section{Regular review for complications and comorbidities in patients with bronchiectasis or chronic suppurative lung disease}

Adults should be reviewed every year and children every 6 months. A multidisciplinary team is recommended, especially when providing initial education. The review should include:

- Assessment of severity, including an assessment of exacerbation frequency, exercise tolerance, signs of right heart failure, pulse oximetry and spirometry. Patients with suspected right heart failure or hypoxia should be referred early.

- Identifying and managing bronchiectasis complications and comorbidities, particularly gastro-oesophageal reflux, reactive airway disease, asthma and chronic obstructive pulmonary disease. Occasionally, patients require assessments for sleepdisordered breathing and cardiac complications.

\section{Priority areas for chronic suppurative lung disease (CSLD) and bronchiectasis in Indigenous Australians living in rural and remote areas}

Research

- Accurate assessment of disease burden.

- Increased understanding of natural history of CSLD and bronchiectasis.

- Evaluation of interventions to:

$>$ prevent progression of CSLD to established bronchiectasis in children;

$>$ reduce frequency and severity of acute exacerbations, including delaying progress of bronchiectasis to uninvolved lung (via antibiotics, mucoactive agents, multidisciplinary care); and

$>$ improve effectiveness of airway clearance techniques and pulmonary rehabilitation.

Service delivery

- Use of spirometry in primary health care, including training and support of staff in supervising and interpreting spirometry.

- Patient access, staff and equipment to perform c-HRCT scans to diagnose bronchiectasis and to conduct bronchoscopy for baseline assessment.

- Training of primary health care doctors, nurses, allied health workers, Indigenous health workers, paediatricians and physicians in diagnosis, management and patient and carer education with regard to CSLD in children and bronchiectasis in both adults and children.

- Access to advice and review by paediatric and adult specialist respiratory services.

- Development of pulmonary rehabilitation programs for adults with bronchiectasis.

- Well defined systems for early identification and referral of patients who may benefit from lung transplantation.

- Development of multidisciplinary teams, particularly including physiotherapists, to help patients and carers in the ongoing management of CSLD and bronchiectasis. Teams should:

$>$ support primary health care providers;

$>$ help with hospitalised patient care; and

$>$ liaise between primary health care and hospital services to optimise ambulatory and inpatient management.

$\mathrm{C}-\mathrm{HRCT}=$ chest high-resolution computed tomography. 


\section{Acknowledgements}

We thank Bilawara Lee, a senior member of the Larrakia Nation, who opened the workshop and welcomed us to her country, where the workshop took place. Anne Chang is supported by the Royal Children's Hospital Foundation and by an NHMRC Practitioner Fellowship.

\section{Competing interests}

Keith Grimwood was previously a member of a Rotavirus Advisory Board in New Zealand and received a research grant from GlaxoSmithKline to study the epidemiology of intussusception in New Zealand. He has also received a research grant from Merck for a rotavirus surveillance study and is currently chair of a Data and Safety Monitoring Committee for phase I and II trials of a meningococcal vaccine for Wyeth. Graeme Maguire receives research funding from the NHMRC, the Office for Aboriginal and Torres Strait Islander Health and the Cooperative Research Centre for Aboriginal Health to facilitate the conduct of research projects on Indigenous Australian respiratory health and disease.

\section{Author details}

Anne B Chang, MPHTM, PhD, FRACP, Paediatric Respiratory Physician, and $\mathrm{Head}^{2}$

Keith Grimwood, MB ChB, FRACP, MD, Paediatric Infectious Diseases

Physician, ${ }^{3}$ and Director of Research, Royal Children's Hospital ${ }^{4}$

Graeme Maguire, MB BS, FRACP, Associate Professor ${ }^{5}$

Paul T King, MB BS, FRACP, PhD, Staff Specialist in Respiratory

Medicine ${ }^{6}$

Peter S Morris, MB BS, FRACP, PhD, Deputy Head, ${ }^{2}$ and Associate

Professor of Child Health ${ }^{7}$

Paul J Torzillo, MB BS, FRACP, Medical Director, ${ }^{8}$ and Senior

Respiratory Physician ${ }^{9}$

1 Department of Respiratory Medicine, Royal Children's Hospital,

Brisbane, QLD

2 Child Health Division, Menzies School of Health Research, Charles

Darwin University, Darwin, NT.

3 Queensland Paediatric Infectious Diseases Laboratory, Royal

Children's Hospital, Brisbane, QLD.

4 Discipline of Paediatrics and Child Health, University of Queensland,

Brisbane, QLD

5 School of Medicine, James Cook University, Cairns, QLD.

6 Department of Medicine and Department of Respiratory Medicine,

Monash Medical Centre, Melbourne, VIC.

7 Northern Territory Clinical School, Flinders University, Darwin, NT.

8 Nganampa Health Council, Alice Springs, NT.

9 Royal Prince Alfred Hospital and University of Sydney, Sydney, NSW.

Correspondence: annechang@ausdoctors.net

\section{References}

1 Standing Committee on Aboriginal and Torres Strait Islander Health and Statistical Information Management Committee 2006. National summary of the 2003 and 2004 jurisdictional reports against the Aboriginal and Torres Strait Islander health performance indicators. Canberra: AlHW, 2006. (AlHW Cat. No. IHW 16.) http://www.aihw.gov.au/publications/index.cfm/title/ 10234 (accessed Jul 2008)

2 Australian Bureau of Statistics. National Aboriginal and Torres Strait Islander Health Survey, 2004-05. Canberra: ABS, 2006. (ABS Cat. No. 4715.0.)

3 Chang AB, Masel JP, Boyce NC, et al. Non-CF bronchiectasis: clinical and HRCT evaluation. Pediatr Pulmonol 2003; 35: 477-483

4 Chang AB, Grimwood K, Mulholland EK, Torzillo PJ; Working Group on Indigenous Paediatric Respiratory Health. Bronchiectasis in Indigenous children in remote Australian communities. Med J Aust 2002; 177: 200-204.

5 King PT, Holdsworth SR, Freezer NJ, et al. Outcome in adult bronchiectasis. COPD 2005; 2: 27-34

6 Keistinen T, Säynäjäkangas O, Tuuponen T, Kivelä SL. Bronchiectasis: an orphan disease with a poorly-understood prognosis. Eur Respir J 1997; 10: 2784-2787.

7 Steinfort DP, Brady S, Weisinger HS, Einsiedel L. Bronchiectasis in Central Australia: a young face to an old disease. Respir Med 2008; 102: 574-578.
8 Chang AB, Bilton D. Non-cystic fibrosis bronchiectasis exacerbations. Thorax 2008; 63: 269-276.

9 Kiechl S, Egger G, Mayr M, et al. Chronic Infections and the risk of carotid atherosclerosis: prospective results from a large population study. Circulation 2001; 103: 1064-1070.

10 Hill SL, Morrison HM, Burnett D, Stockley RA. Short term response of patients with bronchiectasis to treatment with amoxycillin given in standard or high doses orally or by inhalation. Thorax 1986; 41: 559-565.

11 Atkins $D$, Best $D$, Briss PA, et al. Grading quality of evidence and strength of recommendations. BMJ 2004; 328: 1490-1497.

12 Vakil N, van Zanten SV, Kahrilas P, et al; Global Consensus Group. The Montreal definition and classification of gastroesophageal reflux disease: a global evidence-based consensus. Am J Gastroenterol 2006; 101: 1900-1920.

13 Cymbala AA, Edmonds LC, Bauer MA, et al. The disease-modifying effects of twice-weekly oral azithromycin in patients with bronchiectasis. Treat Respir Med 2005: 4: 117-122.

14 King P. Is there a role for inhaled corticosteroids and macrolide therapy in bronchiectasis? Drugs 2007; 67: 965-974.

15 Chang AB, Redding GJ, Everard ML. Chronic wet cough: protracted bronchitis, chronic suppurative lung disease and bronchiectasis. Pediatr Pulmonol 2008; 43: 519-531.

16 Webb WR, Müller NL, Naidich DP. High-resolution CT of the lung. Philadelphia: Lippincott, Williams \& Wilkins, 2001: 467-546.

17 Matsuoka S, Uchiyama K, Shima H, et al. Bronchoarterial ratio and bronchial wall thickness on high-resolution $\mathrm{CT}$ in asymptomatic subjects: correlation with age and smoking. AJR Am J Roentgenol 2003; 180: 513-518.

18 Chang AB, Masel JP, Boyce NC, Torzillo PJ. Respiratory morbidity in central Australian Aboriginal children with alveolar lobar abnormalities. Med J Aust 2003; 178: 490-494.

19 Shoemark A, Ozerovitch L, Wilson R. Aetiology in adult patients with bronchiectasis. Respir Med 2007; 101: 1163-1170.

20 King PT, Holdsworth SR, Freezer NJ, et al. Characterisation of the onset and presenting clinical features of adult bronchiectasis. Respir Med 2006; 100 : 2183-2189.

21 Croxatto OC, Lanari A. Pathogenesis of bronchiectasis; experimental study and anatomic findings. J Thorac Surg 1954; 27: 514-528.

22 Stockley RA. Role of bacteria in the pathogenesis and progression of acute and chronic lung infection. Thorax 1998; 53: 58-62.

23 Valery PC, Torzillo PJ, Mulholland EK, et al. Hospital-based case-control study of bronchiectasis in Indigenous children in Central Australia. Pediatr Infect Dis J 2004: 23: 902-908.

24 Judson MA. Noninvasive Aspergillus pulmonary disease. Semin Respir Crit Care Med 2004; 25: 203-219.

$25 \mathrm{Chan} \mathrm{CH}, \mathrm{Ho} \mathrm{AK}$, Chan RC, et al. Mycobacteria as a cause of infective exacerbation in bronchiectasis. Postgrad Med J 1992; 68: 896-899.

26 Ellerman A, Bisgaard $\mathrm{H}$. Longitudinal study of lung function in a cohort of primary ciliary dyskinesia. Eur Respir J 1997; 10: 2376-2379.

27 Evans DJ, Bara Al, Greenstone M. Prolonged antibiotics for purulent bronchiectasis. Cochrane Database Syst Rev 2007; (2): CD001392.

28 Southern KW, Barker PM, Solis A. Macrolide antibiotics for cystic fibrosis. Cochrane Database Syst Rev 2004; (2): CD002203.

29 Tramper-Stranders GA, Wolfs TF, Fleer A, et al. Maintenance azithromycin treatment in pediatric patients with cystic fibrosis: long-term outcomes related to macrolide resistance and pulmonary function. Pediatr Infect Dis $J$ 2007; 26: 8-12.

30 Rubin BK. Aerosolized antibiotics for non-cystic fibrosis bronchiectasis. J Aerosol Med 2008; Feb 7 [Epub ahead of print].

31 O'Donnell AE, Barker AF, llowite JS, Fick RB. Treatment of idiopathic bronchiectasis with aerosolized recombinant human DNase I. rhDNase Study Group. Chest 1998; 113: 1329-1334.

32 Button BM, Heine RG, Catto-Smith AG, Phelan PD. Postural drainage in cystic fibrosis: is there a link with gastro-oesophageal reflux? J Paediatr Child Health 1998; 34: 330-334.

33 Patterson JE, Bradley JM, Elborn JS. Airway clearance in bronchiectasis: a randomized crossover trial of active cycle of breathing techniques (incorporating postural drainage and vibration) versus test of incremental respiratory endurance. Chron Respir Dis 2004; 1: 127-130.

34 O'Brien C, Guest PJ, Hill SL, Stockley RA. Physiological and radiological characterisation of patients diagnosed with chronic obstructive pulmonary disease in primary care. Thorax 2000; 55: 635-642.

35 Karadag B, Karakoc F, Ersu R, et al. Non-cystic-fibrosis bronchiectasis in children: a persisting problem in developing countries. Respiration 2005; 72 : 233-238.

36 National Health and Medical Research Council. The Australian immunisation handbook. 9th ed. Canberra: NHMRC, 2008.

(Received 26 Dec 2007, accepted 23 Apr 2008) 